Historic, Archive Document

Do not assume content reflects current scientific knowledge, policies, or practices. 



\section{Coolidge Rare Plant Gardens PRICE LIST}

The price is governed by size and rarity of plants. When a quantity of one dozen or more of a single variety is desired, we will be glad to make quotations.

Acacia Cavenia

$\$ 2.50$

Actinidia Chinensis

.1 .00

Anona Cherimoya ...........................00, 5.00

Avocados ................................50, 4.00, 15.00

Azaleas $4.50, \quad 4.00,1.50$

Bauhinia Candida ......................................1.00

Bauhinia Purpurea .................................1.00

Bauhinia Tomentosa ................................. .50

Berberis Dictyota ..................................... .50

Berberis Elegantissima' ............................ .50

Berberis Gagnipani ........................ .50, 2.50

Berberis Pruinosa .................................... .50

Berberis Sargentiana .................... .50, 2.00

Berberis Stenophylla .................... .50, 1.50

Berberis Tricanthophora................ .50, 2.00

Bignonia ........................................................ .75

Bignonia Garrocha ................................. .50

Capparis Spinosa ......................................1.00

Coprosma Cunninghami .............. .50, 2.00

Coprosma Stocki .......................................1.00

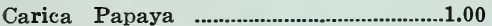

Carissa Grandiflora ....................................1.00

Castanospermum Australe ....................... .50

Catha Edulis ..............................................1.00

Ceanothus Bijou ......................................... .75

Ceanothus Chas. Dietrich ...................... .75

Ceanothus Marie Simon............................ .75

Ceanothus Versailles ................................. .75

Chelone .................................................doz., 1.50

Chinese lemon dwarf.. .50, 1.00, 2.00, 5.00

Cocculus laurifolius ...................... .50, 1.50

Coreopsis ........................................ doz., 1.50

Cotoneaster Heroveana .......................... .50

Cotoneaster Salicifolia ............................. .50

Cuphea ................................................doz., 1.50

Daphne cneorum ......................................2.00

Daphne Odora ...........................................4.00

Eranthemum Pulchellum ........................1.00

Erica Blanda ................................................. .25

Erica Charlesiana .......................................2.5Q

Erica Codonodes Vietchi........................... .25

Erica Hielana (not yet on sale)

Erica Mediterranea ................................... .25

Erica Mediterranea Hybrida .25, .50, 1.50

Erica Melanthera ................... .25, .50, 1.50

Erica Persoluta Alba .......... .25, .50, 1.50

Erica Persoluta Rosea .................... .25, 1.50

Erica Pres. Felix Faure .... .25, .50, 1.50

Erica Regerminans ............... .25, 50, 1.50

Erica Translucens ..................................... .50

Eugenia Hookerii $.25, .75,2.50,5.0010 .00$

Eugenia Jambose ......................................2.50

Eugenia Ugni (Chilean Guava) ............ .50

Feijoa Coolidgeii ................2.50, 5.00, 15.00

Gerbera ......................................doz., .50, 5.00

Grevillea Banksii ..........................2.50, 5.00

Guava .....................................50, 1.00, 1.50

Guava Guinense ....................................... 1.00

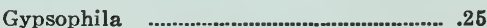

Lepachys Columnaris ........ .50; doz., 5.00

Leptospermum Chapmani ........... .50, 2.50

Leptospermum Flavescans .......... .50, 1.50

Leptospermum Lanigerum .... .25, .50, 1.50

Leptospermum Nicholsi ................ .25, 2.50

Libonia Floribundum ............................... .50

Ligustrum Henryii .... .25, 1.50, 2.50, 5.00

Lonicera Speciosa .......................................1.00

Loropetalum Chinensis ............................2.50

Macadamia Ternifolia .............................5.00

Malus Floribunda purpurea .....................2.00

Malva Chinense ......................................... .50

Myrtus Ralphi ...................................25, $\quad .75$

Olearia Fosteri ............................... .25, 1.00

Olearia Haasti ........................................1.00

Osteomeles anthyllidifolia ............ .50, 1.50

Osteomeles Schwerina .................. .50, 1.50

Passiflora Edulis or Grenadilla ............ .50

Pavonia Hastata ...................................... .50

Philadelphis Sempervirens .................... .50

Physostegia ........................................doz., 1.50

Pittosporum Floribundum................ .25, 1.50

Plumeria or Frangipani .........................5.00

Podocarpus Elongatus ............................1.00

Polystichium Plumosum Densum ........ .75

Prunus Campanulata ..............................5.00

Punica Granatum Nana .................. .25, .50

Pyracantha Formosiana ........ .25, .75, 2.50

Pyrus Kawakami .....................................1.00

Quillaja Saponaria .................................1.00

Rapheolepis Delacourei .............................1.00

Rangpur Lime ...............................................50

Rhus Lancea ................................................1.50

Salvia Pitcheri ........................................... .50

Sarcoccoccoa Ruscifolia .............................1.00

Shasta Daisy Pasadena .... .50; doz., 4.00

Sphaeralcea Vitifolia .....................1.00, 2.50

Stranvesia Undulatum ............................. .75

Tibouchina Semidecandra ...................... 2.00

Tricuspidaria Dependens ........................5.00

Ulmus Parvifolius ......................... .50, 1.50

Viburnum Rectangulum ................. .50, 2.00

Viburnum Rhytidophyllum ...................2.00

ROSES-

Belle of Portugal ......................\$1.50 to 5.00

Constance ......................................................1.50

Cheerful ...............................................................50

Duchess of Luxembourg ..........................2.00

Golden Ophelia .......................................50

Golden Emblem ..........................................2.00

Isobel ..................................................................50

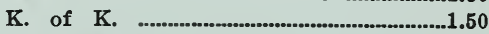

Los Angeles .................................................1.00

Marcia Coolidge .........................................2.00

Mrs. Chas. Bell ......................... .75 to 1.50

Rose Marie .................................................1.50

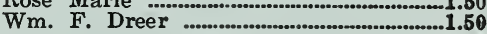


